High-mass X-ray binaries: illuminating the passage from massive

binaries to merging compact objects

Proceedings IAU Symposium No. 346, $2018 \quad$ C) 2018 International Astronomical Union

A.C. Editor, B.D. Editor \& C.E. Editor, eds. DOI: 00.0000/X000000000000000X

\title{
On the origin of supergiant fast X-ray transients
}

\author{
Swetlana Hubrig ${ }^{1}$, Lara Sidoli ${ }^{2}$, Konstantin A. Postnov ${ }^{3}$, \\ Markus Schöller ${ }^{4}$, Alexander F. Kholtygin ${ }^{5}$, and Silva P. Järvinen ${ }^{1}$ \\ ${ }^{1}$ Leibniz-Institut für Astrophysik Potsdam (AIP), An der Sternwarte 16, 14482 Potsdam, \\ Germany, email: shubrig@aip.de \\ ${ }^{2}$ INAF, Istituto di Astrofisica Spaziale e Fisica Cosmica, Via E. Bassini 15, 20133 Milano, Italy \\ ${ }^{3}$ Sternberg Astronomical Institute, Moscow M.V. Lomonosov State University, \\ 119234 Moscow, Russia \\ ${ }^{4}$ European Southern Observatory, Karl-Schwarzschild-Str. 2, 85748 Garching, Germany \\ ${ }^{5}$ Saint-Petersburg State University, Universitetskij pr. 28, 198504 Saint-Petersburg, Russia
}

\begin{abstract}
A fraction of high-mass X-ray binaries are supergiant fast X-ray transients. These systems have on average low X-ray luminosities, but display short flares during which their Xray luminosity rises by a few orders of magnitude. The leading model for the physics governing this X-ray behaviour suggests that the winds of the donor OB supergiants are magnetized. In agreement with this model, the first spectropolarimetric observations of the SFXT IGR J112155952 using the FORS 2 instrument at the Very Large Telescope indicate the presence of a kG longitudinal magnetic field. Based on these results, it seems possible that the key difference between supergiant fast X-ray transients and other high-mass X-ray binaries are the properties of the supergiant's stellar wind and the physics of the wind's interaction with the neutron star magnetosphere.
\end{abstract}

Keywords. stars: magnetic fields, stars: individual (IGR J08408-4503, IGR J11215-5952), (stars:) supergiants, (stars:) binaries: general, X-rays: stars

\section{Introduction}

Among the bright X-ray sources in the sky, a significant number contain a compact object (either a neutron star or a black hole) accreting from the wind of a companion star with a mass above $10 M_{\odot}$. Such systems are called high-mass X-ray binaries (HMXBs). They are young (several dozen million years old) and can be formed when one of the initial binary members loses a significant part of its mass through stellar wind or mass transfer before a first supernova explosion occurs (van den Heuvel \& Heise 1972).

Supergiant Fast X-ray Transients (SFXTs) are a subclass of HMXBs associated with early-type supergiant companions, and characterized by sporadic, short and bright $\mathrm{X}_{-}$ ray flares reaching peak luminosities of $10^{36}-10^{37} \mathrm{erg} \mathrm{s}^{-1}$ and typical energies released in bright flares of about $10^{38}-10^{40} \mathrm{erg}$ - see the review by Sidoli (2017) for more details. Their X-ray spectra in outburst are very similar to accreting pulsars in HMXBs. In fact, half of them have measured neutron star spin periods similar to those observed from persistent HMXBs (Shakura et al. 2015, Martinez-Nunez et al. 2017). The physical mechanism driving their transient behavior, probably related to the accretion of matter from the supergiant wind by the compact object, has been discussed by several authors and is still a matter of debate. The leading model for the existence of SFXTs invokes their different wind properties and magnetic field strengths that lead to distinctive accretion regimes (Shakura et al. 2012, Postnov et al. 2015, Shakura et al. 2015, Shakura \& Postnov 2017). The SFXTs' behaviour can be explained by sporadic capture of magnetized stellar 


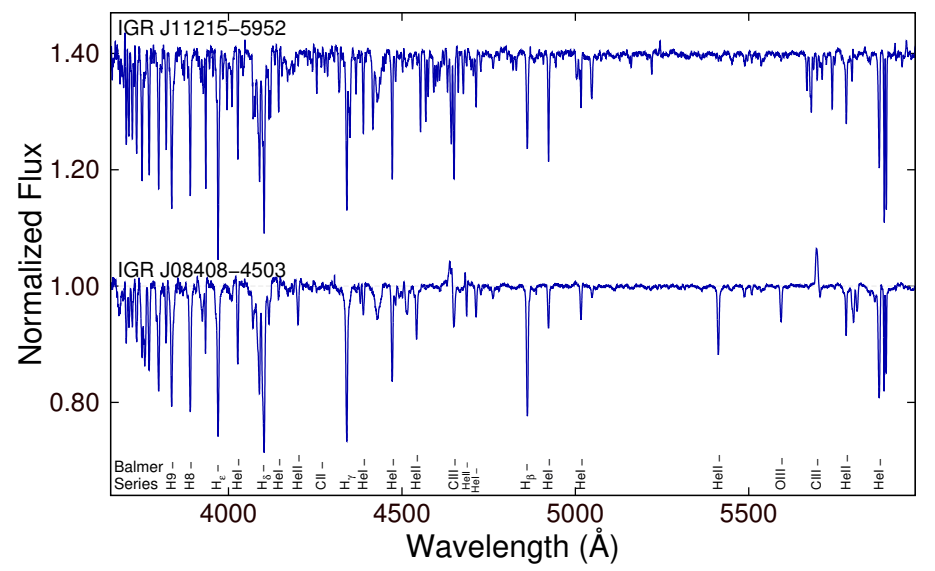

Figure 1. Normalised FORS 2 Stokes $I$ spectra of IGR J08408-4503 and IGR J11215-5952. Well known spectral lines are indicated at the bottom. The spectrum of IGR J11215-5952 was vertically offset by 0.4 for clarity.

wind. The effect of the magnetic field carried by the stellar wind is twofold: first, it may trigger rapid mass entry to the magnetosphere via magnetic reconnection in the magnetopause (a phenomenon that is well known in the dayside of Earth's magnetosphere), and secondly, the magnetized parts of the wind (magnetized clumps with a tangent magnetic field) have a lower velocity than the non magnetised parts (or the parts carrying the radial field; Shakura et al. 2015). The model predicts that a magnetized clump of stellar wind with a magnetic field strength of a few $10 \mathrm{G}$ triggers sporadic reconnection, allows accretion, and results in an X-ray flare. Typically, the neutron star orbital separation is a few $R_{*, \mathrm{RSG}}$. Thus, the expected required magnetic field on the stellar surface is of the order of $100-1000 \mathrm{G}$.

\section{Magnetic field measurements}

To investigate the magnetic nature of SFXTs, we recently observed the two optically brightest targets, IGR J08408-4503 $\left(P_{\text {orb }}=9.5 \mathrm{~d}\right)$ and IGR J11215-5952 $\left(P_{\text {orb }}=165 \mathrm{~d}\right)$, using the FOcal Reducer low dispersion Spectrograph (FORS 2; Appenzeller et al. 1998) mounted on the $8 \mathrm{~m}$ Antu telescope of the Very Large Telescope in spectropolarimetric mode. No significant magnetic field was measured in the spectra of IGR J08408-4503, with the highest value $\left\langle B_{\mathrm{z}}\right\rangle_{\text {hyd }}=-184 \pm 97 \mathrm{G}$ at a significance level of $1.9 \sigma$. On the other hand, a definite magnetic field detection was achieved for IGR J11215-5952 in 2016 December with $\left\langle B_{\mathrm{z}}\right\rangle_{\text {hyd }}=416 \pm 110 \mathrm{G}$ with a significance at the $3.8 \sigma$ level. The measurement obtained in 2016 May yielded $\left\langle B_{\mathrm{z}}\right\rangle_{\text {hyd }}=-978 \pm 308 \mathrm{G}$ at a significance level of $3.2 \sigma$ (Hubrig et al. 2018). The spectral appearance of IGR J08408-4503 and IGR J11215-5952 in the FORS 2 spectra is presented in Fig. 1

In Fig. 2, we present Stokes $V$ spectra of IGR J11215-5952 obtained on these two nights in the spectral region around the $\mathrm{H} \beta$ line. For best visibility of the Zeeman features, we overplot the Stokes $V$ spectra of IGR J11215-5952 with the Stokes $V$ spectra of the two well-known magnetic early B-type stars HD 96446 and $\xi^{1}$ CMa.

Regarding the significance of the magnetic field detections in massive stars at significance levels around $3 \sigma$, we note that the two clearly magnetic Of?p stars HD 148937 and CPD $-28^{\circ} 5104$ have been for the first time detected as magnetic in our FORS 2 observations at significance levels of $3.1 \sigma$ and $3.2 \sigma$, respectively (Hubrig et al. 2008, Hubrig et 

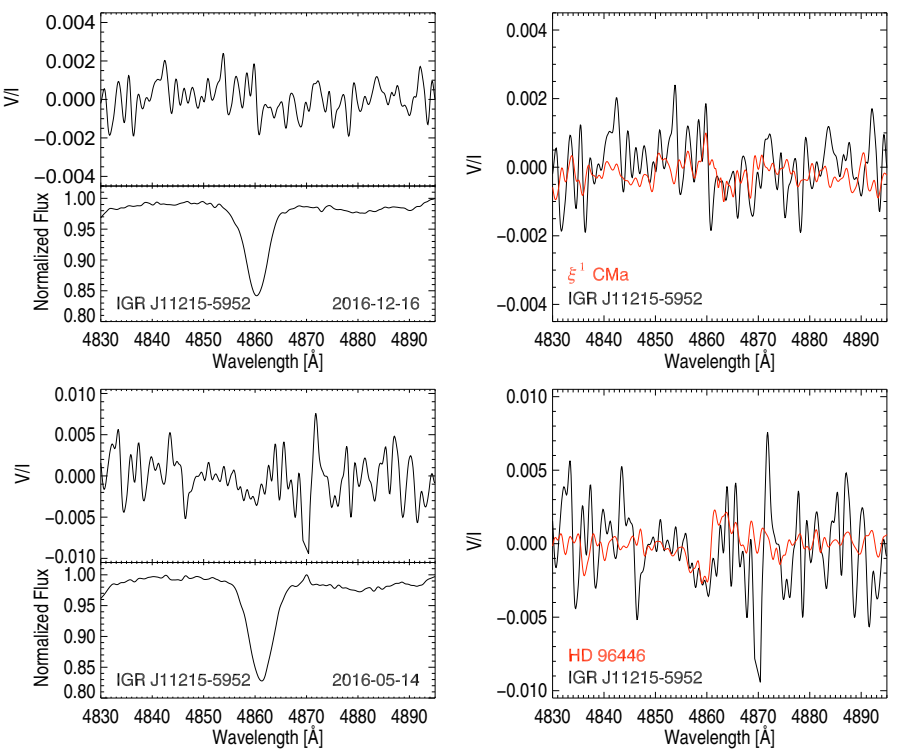

Figure 2. Left panel: Stokes $V$ and Stokes $I$ spectra of IGR J11215-5952 in the spectral region around the $\mathrm{H} \beta$ line at two different epochs. Right panel: Stokes $V$ spectra of IGR J11215-5952 overplotted with the Stokes $V$ spectra of the two well-known magnetic early-B type stars $\xi^{1} \mathrm{CMa}$ $\left(\left\langle B_{\mathrm{z}}\right\rangle_{\mathrm{hydr}}=360 \pm 49 \mathrm{G}\right)$ and HD $96446\left(\left\langle B_{\mathrm{z}}\right\rangle_{\mathrm{hydr}}=-1590 \pm 74 \mathrm{G}\right)$ for best visibility of the Zeeman features.

al. 2011). The detection of a magnetic field in IGR J11215-5952 at significance levels of $3.2 \sigma$ and $3.8 \sigma$ indicates that this target likely possesses a $\mathrm{kG}$ magnetic field. Although no significant magnetic field was measured in the spectra of IGR J08408-4503, due to the presence of distinct Zeeman features in its spectra it appears still valuable to obtain additional spectropolarimetric observations on other epochs corresponding to different orbital phases.

\section{Discussion}

Our spectropolarimetric observations of IGR J11215-5952 revealed the presence of a magnetic field on two occasions. This target is the only SFXT where strictly periodic Xray outbursts have been observed, repeating every $164.6 \mathrm{~d}$ (Sidoli et al. 2006, Sidoli et al. 2007, Romano et al. 2009). To explain these short periodic outbursts, Sidoli et al. (2007) proposed that they are triggered by the passage of the neutron star inside an equatorial enhancement of the outflowing supergiant wind, focussed on a plane inclined with respect to the orbit. This configuration of the line-driven stellar wind might be magnetically channeled (ud-Doula \& Owocki 2002). The effectiveness of the stellar magnetic field in focussing the wind is indicated by the wind magnetic confinement parameter $\eta$ defined as $\eta=B_{*}^{2} R_{*}^{2} /\left(\dot{M}_{\mathrm{w}} v_{\infty}\right)$, where $\mathrm{B}_{*}$ is the strength of the magnetic field at the surface of the supergiant, $R_{*}$ is the stellar radius $\left(R_{*}=40 R_{\odot}\right), v_{\infty}$ is the wind terminal velocity $\left(v_{\infty}=1200 \mathrm{~km} \mathrm{~s}^{-1}\right)$ and $\dot{M}_{\mathrm{w}}$ is the wind mass loss rate $\left(\dot{M}_{\mathrm{w}}=10^{-6} \mathrm{M}_{\odot} \mathrm{yr}^{-1}\right.$; Lorenzo et al. 2014). Adopting $\mathrm{B}_{*} \geqslant 0.7 \mathrm{kG}$ at the magnetic equator, we estimate $\eta \geqslant 500$, implying a wind confinement, up to an Alfvén radius $R_{A}=\eta^{1 / 4} R_{*} \geqslant 4.73 R_{*}$ (ud-Doula \& Owocki 2002). This radial distance is compatible with the orbital separation at periastron in IGR J11215-5952, where the orbital eccentricity is high ( $e>0.8$; Lorenzo et al. 2014). The measured magnetic field strength in IGR J11215-5952 reported here for the first 
time is high enough to channel the stellar wind on the magnetic equator, supporting the scenario proposed by Sidoli et al. (2007) to explain the short periodic outbursts in this SFXT.

Because of the faintness of SFXTs - most of them have a visual magnitude $m_{V} \geqslant 12$, up to $m_{V} \geqslant 31$ (Sidoli 2017, Persi et al. 2015) - no high-resolution spectropolarimetric observations were carried out for these objects so far and the presented FORS 2 observations are the first to explore the magnetic nature of the optical counterparts. Future spectropolarimetric observations of a representative sample of SFXTs are urgently needed to be able to draw solid conclusions about the role of magnetic fields in the wind accretion process and to constrain the conditions that enable the presence of magnetic fields in massive binary systems.

\section{References}

Appenzeller, I., Fricke, K., Fürtig, W., Gässler, W., Häfner, R., Harke, R., Hess, H.-J., Hummel, W., Jürgens, P., Kudritzki, R.-P., Mantel, K.-H., Meisl, W., Muschielok, B., Nicklas, H., Rupprecht, G., Seifert, W., Stahl, O., Szeifert, T., \& Tarantik, K. 1998, The ESO Messenger, 94, 1

Hubrig, S., Schöller, M., Schnerr, R.S., González, J.F., Ignace, R., \& Henrichs, H. 2008, A\& A, 490, 793

Hubrig, S., Schöller, M., Kharchenko, N.V., Langer, N., de Wit, W.J., Ilyin, I., Kholtygin, A.F., Piskunov, A.E., Przybilla, N., \& Magori Collaboration 2011, A\&A A, 528, A151

Hubrig, S., Sidoli, L., Postnov, K., Schöller, M., Kholtygin, A.F., Järvinen, S.P., \& Steinbrunner, P. 2018, MNRAS, 474, L27

Lorenzo, J., Negueruela, I., Castro, N., Norton, A.J., Vilardell, F., \& Herrero, A. 2014, A\&GA, 562, A 18

Martinez-Nunez, S., Kretschmar, P., Bozzo, E., Oskinova, L.M., Puls, J., Sidoli, L., Sundqvist, J.O., Blay, P., Falanga, M., Fürst, F., Gímenez-García, A., Kreykenbohm, I., Kühnel, M., Sander, A., Torrejón, J.M., \& Wilms, J. 2017, SSRv, 212, 59

Persi, P., Fiocchi, M., Tapia, M., Roth, M., Bazzano, A., Ubertini, P., \& Parisi, P. 2015, AJ, 150, 21

Postnov, K.A., Gornostaev, M.I., Klochkov, D., Laplace, E., Lukin, V.V., \& Shakura, N.I. 2015, MNRAS, 452, 1601

Romano, P., Sidoli, L., Cusumano, G., Vercellone, S., Mangano, V., \& Krimm, H.A. 2009, ApJ, 696, 2068

Shakura, N., Postnov, K., Sidoli, L., \& Paizis, A. 2014, MNRAS, 420, 216

Shakura, N.I., Postnov, K.A., Kochetkova, A.Yu., Hjalmarsdotter, L., Sidoli, L., \& Paizis, A. 2015. ARep, 59, 645

Shakura, N., \& Postnov, K. 2017, Accretion Processes in Cosmic Sources, September 5-10, 2016, St-Petersburg, also: arXiv:1702.03393

Sidoli, L., Paizis, A., \& Mereghetti, S. 2006, A\&A, 450, L9

Sidoli, L., Romano, P., Mereghetti, S., Paizis, A, Vercellone, S., Mangano, V., \& Götz, D. 2007, $A \& A, 476,1307$

Sidoli, L. 2017, Proc. of the "XII Multifrequency Behaviour of High Energy Cosmic Sources Workshop", 12-17 June, 2017 Palermo, Italy, 52

van den Heuvel, E.P.J., \& Heise, J. 1972, Nature Physical Science, 239, 67

ud-Doula, A., \& Owocki, S.P. 2002, ApJ, 576, 413 